\title{
JOIN trial: treatment outcome and recovery status of peripheral sensory neuropathy during a 3-year follow-up in patients receiving modified FOLFOX6 as adjuvant treatment for stage II/III colon cancer
}

\author{
Takayuki Yoshino $^{1} \cdot$ Masahito Kotaka $^{2} \cdot$ Katsunori Shinozaki $^{3} \cdot$ Tetsuo Touyama $^{4} \cdot$ Dai Manaka $^{5} \cdot$ Takanori Matsui $^{6}$. \\ Kiyoshi Ishigure $^{7}$. Junichi Hasegawa ${ }^{8} \cdot K$ eiji Inoue ${ }^{9}$. Yoshinori Munemoto ${ }^{10}$. Akinori Takagane ${ }^{11}$. \\ Hiroshi Ishikawa $^{12} \cdot$ Hideyuki Ishida $^{13}$ - Yutaka Ogata ${ }^{14} \cdot K_{\text {Koji Oba }}{ }^{15}$ • Koichi Goto ${ }^{16}$. Junichi Sakamoto ${ }^{17}$. \\ Yoshihiko Maehara ${ }^{18}$. Atsushi Ohtsu ${ }^{1}$
}

Received: 26 April 2019 / Accepted: 4 September 2019 / Published online: 23 September 2019

(c) The Author(s) 2019

\begin{abstract}
Purpose Adjuvant FOLFOX therapy is an established standard-of-care for resected colon cancer. Peripheral sensory neuropathy (PSN) is regarded as the major toxicity issue related to FOLFOX therapy. There have been a few reports on the recovery status from PSN thereafter. JOIN trial investigated the tolerability and efficacy of adjuvant modified FOLFOX6 (mFOLFOX6) in Japanese patients with stage II/III colon cancer.

Methods Twelve cycles of mFOLFOX6 were given to patients with stage II/III curatively resected colon cancer. Treatment outcomes, including disease-free survival (DFS), relapse-free survival (RFS), overall survival (OS), and recovery status of PSN during 3-year follow-up, were investigated.

Results Of the 882 patients enrolled from 2010 to 2012, 864 were eligible for the efficacy analyses. Three-year DFS, RFS, and OS were favorable in 92.1, 92.8, and 97.4\% of stage II patients; 76.4, 77.9, and 93.8\% of stage IIIA/B; and 61.6, 62.7, and $85.9 \%$ of stage IIIC, respectively. The cumulative incidence of PSN during treatment was $47.8 \%$ in grade 1 (G1), 30.3\% in G2, and 5.8\% in G3. For those with G3 PSN during treatment, there was gradual recovery in $1.1 \%$ of patients at 12 months after enrollment, $0.5 \%$ at 24 months, and $0.2 \%$ at 36 months. However, G1 or G2 residual PSN after 3 years was observed in $21.0 \%(18.7 \%, \mathrm{G} 1 ; 2.3 \%, \mathrm{G} 2)$.

Conclusions Adjuvant mFOLFOX6 therapy was effective and well tolerated in patients with stage II/III colon cancer. Most patients recovered from G3 PSN related to oxaliplatin, but approximately $20 \%$ of patients had G1 or G2 PSN at 3-year follow-up.
\end{abstract}

Keywords Modified FOLFOX6 · Long-term peripheral sensory neuropathy $\cdot$ Oxaliplatin $\cdot$ Colon cancer $\cdot$ Efficacy

\section{Introduction}

Six months of adjuvant oxaliplatin-based 5-fluorouracil (5-FU), leucovorin and oxaliplatin (FOLFOX) chemotherapy following surgery is the standard care for patients with stage III colon cancer as well as for patients with high-risk

Electronic supplementary material The online version of this article (https://doi.org/10.1007/s00280-019-03957-5) contains supplementary material, which is available to authorized users.

Takayuki Yoshino

tyoshino@east.ncc.go.jp

Extended author information available on the last page of the article stage II colon cancer who have risk factors for recurrence that are associated with a relatively poor prognosis, such as T4 status, poorly differentiated histology, vascular invasion, ileus, $<12$ lymph nodes examined, and neural invasion, as recommended by several treatment guidelines [1-3].

FOLFOX4 regimen has proven efficacy in the adjuvant treatment of resected stage II and stage III colon cancers, as demonstrated by the pivotal MOSAIC $[4,5]$ and MASCOT [6] trials conducted in Western and Asian patient populations, respectively. More recently, FOLFOX4 has frequently been substituted by the modified FOLFOX6 (mFOLFOX6) regimen in the adjuvant setting [7, 8], which is easier to administer, and mFOLFOX6 has been shown to be tolerable in the adjuvant treatment of Japanese 
patients with resected stage II and stage III colon cancer in the JOIN (JFMC41-1001-C2) trial [9].

Many patients, however, develop peripheral sensory neuropathy (PSN) by the standard 6 months, 12 cycles of mFOLFOX6 administration, leading to treatment discontinuations [10-14]. PSN can be troublesome in daily life and problems usually persist long after treatment has finished. In the key trials of adjuvant oxaliplatin-based therapy, the incidences of grade $\geq 3$ PSN due to FOLFOX4 therapy during treatment were $12.4 \%$ and $5.7 \%$ for the MOSAIC and Asian MASCOT trials, respectively [4-6]. In the adjuvant US NSABP C-08 trial [7] and Japanese JOIN trial [9] in which patients received the mFOLFOX6 regimen, the incidences of grade $\geq 3 \mathrm{PSN}$ at the end of the studies were $14.4 \%$ and $5.8 \%$, respectively. During a 3 -year follow-up in patients receiving FOLFOX4 in the MOSAIC trial [5], the recovery status of PSN for any grade and grade 3 PSNs was reported as having a frequency of 18.1 and $0.6 \%$, respectively. No trial has revealed the PSN recovery rate from mFOLFOX6, particularly in Asian patients. In addition, 6 months of FOLFOX treatment even in the post-IDEA collaboration era is a standard adjuvant treatment in patients with curatively resected stage II/III colon cancer [3]. The results of the JOIN trial for safety during the treatment course and the treatment compliance have been reported elsewhere [9]. Here, we report the treatment outcomes including diseasefree survival (DFS), relapse-free survival (RFS), overall survival (OS), and recovery rate from PSN during a 3-year follow-up.

\section{Patients and methods}

\section{Study design}

The study design of the JOIN study has been reported previously [9]. Briefly, the JOIN trial is a single-arm, multicenter, large-scale clinical trial across Japan to confirm the tolerability of adjuvant mFOLFOX6 in patients with curatively resected stage II/III colon cancer (UMIN ID: UMIN000004443).

\section{Treatments}

The study treatment was mFOLFOX6 therapy (L-OHP, $85 \mathrm{mg} / \mathrm{m}^{2}$; 1-LV, $200 \mathrm{mg} / \mathrm{m}^{2}$; 5-FU bolus, $400 \mathrm{mg} / \mathrm{m}^{2}$; and 5-FU infusion, $2400 \mathrm{mg} / \mathrm{m}^{2}$ ), with a total of 12 courses being administered at 2-week intervals. Further chemotherapy was not given until recurrence after completion of the scheduled therapy.

\section{Endpoints}

The primary endpoints were the incidence of PSN persisting for $\geq 8$ days that interfered with activities of daily living and with an incidence of $\geq$ grade 3 allergic reactions/anaphylaxis (AR). Secondary endpoints were DFS, RFS, OS, time to treatment failure, adverse events (AEs), comparison of PSN between patients with or without receiving prophylactic therapy, recovery status of PSN during the 3-year followup period, the treatment completion rate, the relative dose intensity (RDI), and the number of lymph-node metastases and number of dissected lymph nodes in relation to the prognosis. DFS was defined as the time from enrollment to relapse, secondary primary colorectal cancer, or death, whichever occurred first. RFS was defined as the time from enrollment to relapse, or death, whichever occurred first. OS was measured from the time of enrollment until death from any cause.

AEs were evaluated according to the Common Terminology Criteria for Adverse Events (CTCAE) Version 4.0. However, PSN was evaluated by following the NCI-CTC Version 1.0, 2.0 and CTCAE Version 3.0.

\section{Statistical analysis}

Descriptive statistics were calculated as the number of patients and percentage for categorical baseline characteristics and mean with a range for continuous baseline characteristics. Three-year DFS, RFS, and OS rates were estimated using the Kaplan-Meier method. Greenwood's formula was applied for calculation of the $95 \%$ confidence interval (95\% CI) of the 3-year DFS, RFS, and OS. The multivariate Cox proportional hazard model was applied to evaluate the prognostic value of patient baseline characteristics for DFS, RFS, and OS. Covariates were selected using the stepwise method with an inclusion criterion of $p<0.20$. The proportion of recovery status of PSN during the 3-year follow-up period was calculated by the number of PSN in the total efficacy population at each follow-up. All statistical analyses were performed using SAS Version 9.3 (SAS Institute, Cary, NC, USA).

\section{Results}

\section{Patient population and baseline characteristics}

Between November 2010 and March 2012, 882 patients were enrolled at 198 institutions. Among these 882 patients, 11 were ineligible, as previously reported [9]. Of the remaining 871 eligible patients, 864 patients $(98.0 \%)$ for whom 
the treatment status was fixed with a median follow-up of 3 years as of October 30, 2015 via an electronic data capture system (Viedoc ${ }^{\circledR}$, PCG Solutions, Uppsala, Sweden) with central monitoring were included in the efficacy analysis (Fig. 1). The characteristics of these patients are shown in Table 1. Baseline patient characteristics were as follows: median age, 64 years; male, 53.8\%; PS 0, 93.8\%; stage II/IIIA/IIIB/IIIC by TNM Classification, 7th edition, 18.5/7.3/52.5/21.6\%; and lymph nodes examined, $<12 / \geq 12 /$ unknown: $17.2 / 82.5 / 0.2 \%$, respectively.

\section{Treatment outcome}

Three-year DFS, RFS, and OS in the overall efficacy population were $76.1 \%$ (95\% CI 73.0-78.8), 77.3\% (95\% CI 74.3-80.0), and 92.7\% (95\% CI 90.7-94.3), respectively (Fig. 2). Favorable 3-year DFS, RFS, and OS were 92.1, 92.8, and $97.4 \%$ in stage II patients, while these were 76.4 , 77.9 , and $93.8 \%$ in stage IIIA/B; and 61.6, 62.7, and $85.9 \%$ in stage IIIC, respectively (Fig. 3). The main recurrent sites were liver (7.6\%), lung (7.3\%), and lymph nodes $(5.2 \%)$. In multivariate Cox regression analysis, tumor histology, venous invasion, and lymph-node metastatic ratio were statistically significant prognostic factors for DFS, RFS, and OS (Table 2), while the tumor location was not significant, although OS in left-sided primary tumors was better than that in right-sided ones with statistical non-significance (Supplementary Fig. 1).
Table 1 Patient characteristics

\begin{tabular}{ll}
\hline$n(\%)$ & 864 \\
Male/female & $465 / 399(53.8 / 46.2)$ \\
Median age (range) & $64(21-83)$ \\
PS: & $810 / 54$ \\
$0 / 1$ & $(93.8 / 6.2)$ \\
Stage (TNM 7th) : & $100 / 34 / 26 / 63 / 454 / 187$ \\
IIA/IIB/IIC/IIIA/IIIB/IIIC & $(11.6 / 3.9 / 3.0 / 7.3 / 52.5 / 21.6)$ \\
Number of lymph nodes examined & $2 / 149 / 713$ \\
Unknown/1-11/ $\geq 12$ & $(0.2 / 17.2 / 82.5)$ \\
Number of positive lymph nodes & $160 / 424 / 280$ \\
$0 / 1-3 / \geq 4$ & $(18.5 / 49.1 / 32.4)$ \\
\hline
\end{tabular}

${ }^{\mathrm{a}} \mathrm{TNM}$ classification of malignant tumors, 7 th edition

\section{Recovery status of PSN}

The cumulative incidence of PSN during treatment was $47.8 \%$ grade $1,30.3 \%$ grade 2 , and $5.8 \%$ grade 3 , respectively (Fig. 4). Grade 3 PSN appeared to gradually recover from $5.8 \%$ to $1.1 \%, 0.5 \%$, and $0.2 \%$ at 12 months, 24 months, and 36 months after enrollment, respectively. However, grade 1 or grade 2 PSNs after 3-year follow-up were observed in $21.0 \%$ of patients $(18.7 \%$ in grade 1 and $2.3 \%$ in grade 2). The transition from each grade of PSN during study treatment (from grade 1 , grade 2 , and grade 3 ) is shown in Supplementary Fig. 2.
Fig. 1 CONSORT diagram. Between November 2010 and March 2012, 882 patients were enrolled at 198 institutions. Among these 882 patients, 11 were ineligible, as previously reported. Of the remaining 871 eligible patients, 864 patients (98.0\%) for whom the treatment status was fixed were included in the efficacy analysis

\section{Consort Flow Diagram}

Enrollment Period from Nov 2010 to Mar 2012

\begin{tabular}{c}
\hline Assigned patient population \\
$(n=882)$ \\
\hline
\end{tabular}

Ineligible $(n=11)$ -Histological stage not adequate $(n=2)$ -Uncontrolled diabetes $(n=1)$ -Inappropriate registration $(n=2)$ -PSN $(n=1)$

-Past history of malignancy $(n=5)$

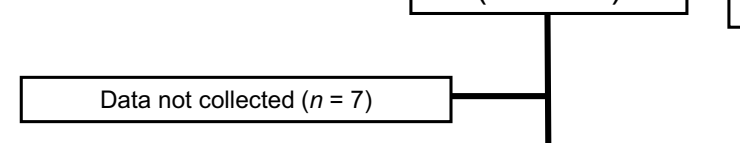



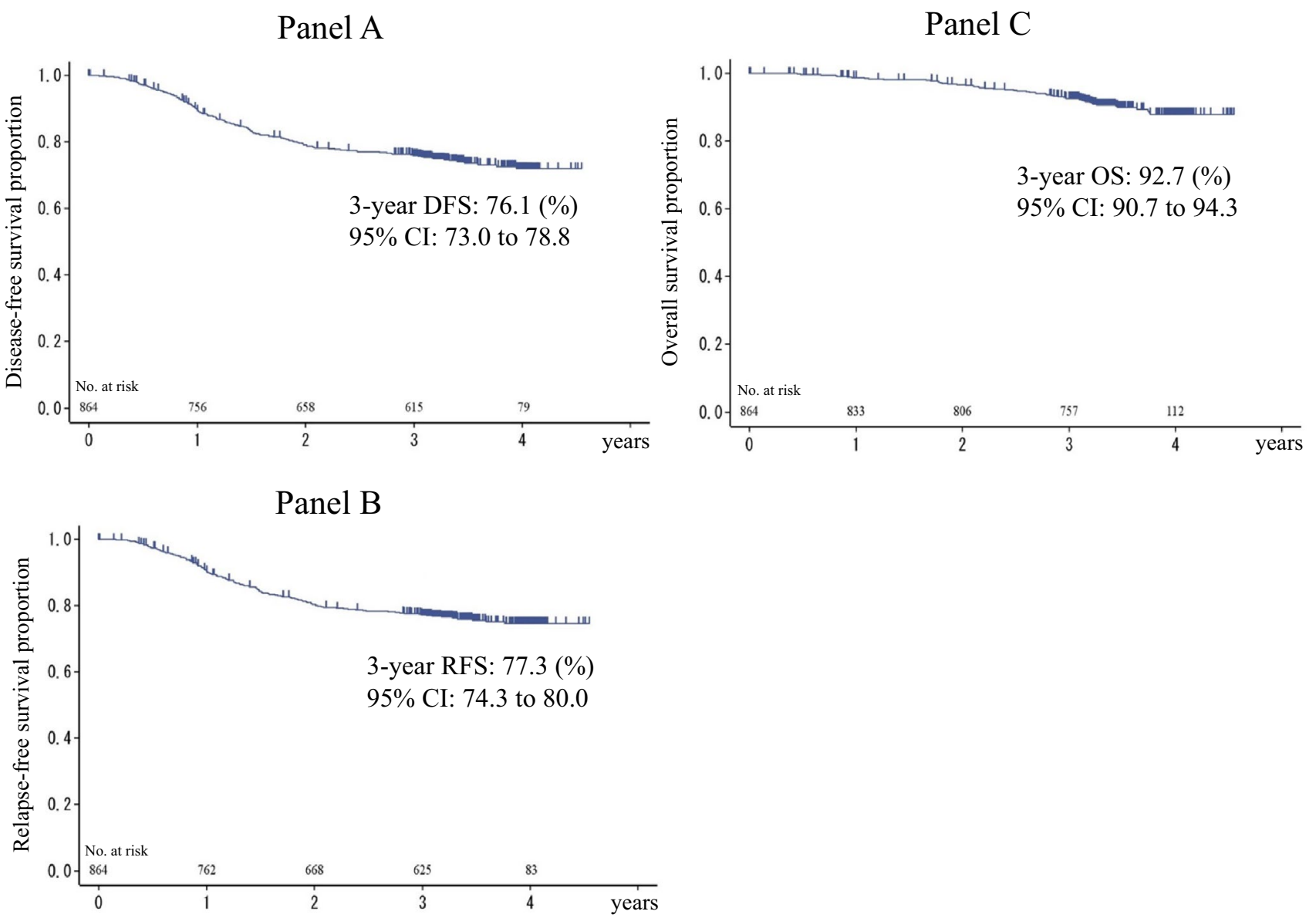

Fig. 2 Kaplan-Meier curves for a DFS, b RFS, and c OS in the overall population. DFS disease-free survival, RFS relapse-free survival, $O S$ overall survival, 95\% CI 95\% confidence interval

\section{Discussion}

The JOIN trial results confirm the efficacy and safety of adjuvant mFOLFOX6 in over 800 patients with stage II/ III curatively resected colon cancer among approximately 200 community hospitals in Japan. Approximately $6 \%$ of Asian patients only showed grade 3 or higher PSN in the JOIN and MASCOT, while $12-14 \%$ patients did in the Western trials such as MOSAIC and NSABP C-08 [5-9].

The PSN during treatment was the highest and gradually recovered during the 3 -year follow-up period. In addition, it was confirmed that approximately $20 \%$ of patients had grade 1 or grade 2 PSN from the 3 -year PSN followup, which is consistent with that of FOLFOX4 [5]. PSN caused by oxaliplatin, either FOLFOX4 or mFOLFOX6, is a common issue worldwide. However, the remaining PSN may be influenced by factors other than oxaliplatin. In the NSABP C-07 trial [15] comparing weekly bolus fluorouracil and leucovorin (FL) therapy with or without oxaliplatin, the PSN for some patients (less than 5\%) remained in the FL group at 12 months of follow-up, suggesting that the PSN may occur due to factors other than oxaliplatin.

Three-year DFS and OS in the JOIN trial were 76.1 and $92.7 \%$, respectively. Stratified by stage, 3 -year DFS and OS were 92.1 and $97.4 \%$ in stage II, 76.4 and $93.8 \%$ in stage IIIA/B, 61.6, and $85.9 \%$ in stage IIIC, respectively. Threeyear DFS in other recent clinical trials conducted in Japan for stage II/III or stage III colon cancer were reported to range from 70.0 to $86.2 \%$, while those in 3-year OS were reported to range from 92.7 to $98.0 \%$, which is consistent with those in the JOIN trial (Supplementary Table) [16-22].

Based on the results of Japanese clinical trials including the JOIN trial, the survival rate of patients with stage II/III colon cancer appeared to be relatively higher in Japan than in Western countries (Supplementary Table) [16-22]. This difference might be largely due to the lower percentage of patients with $<12$ nodes examined as well as the Japanese D3 lymph-node dissection procedure, given that the number of lymph nodes examined after surgical resection was shown to correlate with survival [23]. In fact, the percentage of patients with $<12$ nodes examined in Japanese trials 


\section{Panel A}

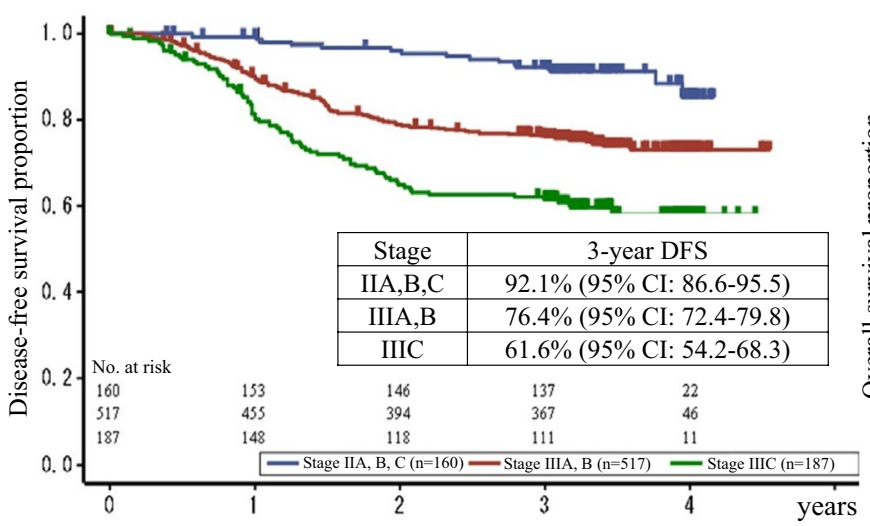

Panel B

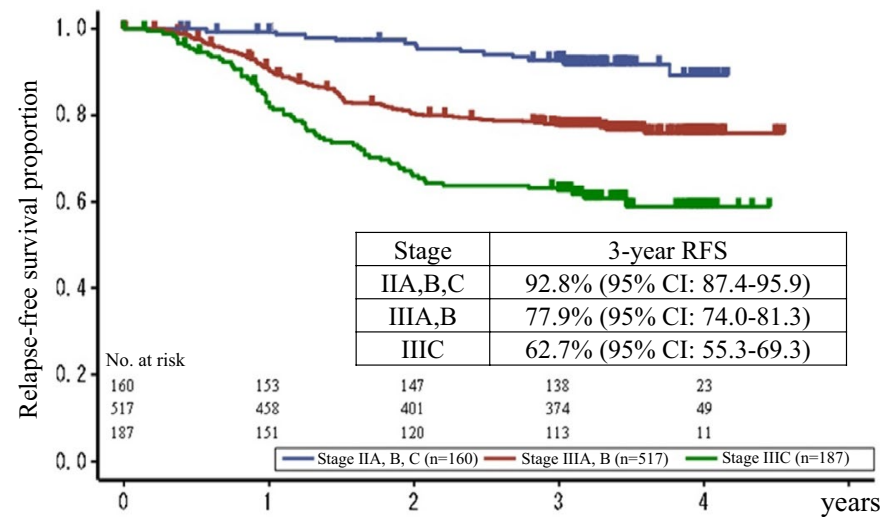

\section{Panel C}

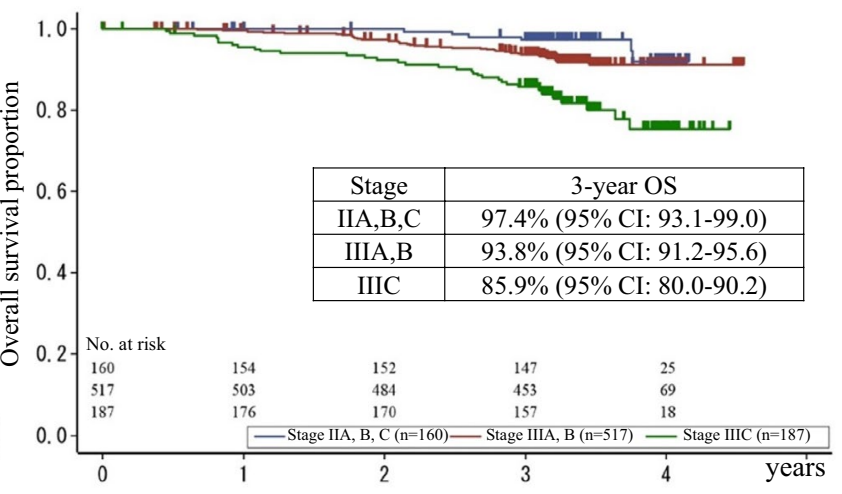

Fig. 3 Kaplan-Meier curves for a DFS, b RFS and $\mathbf{c}$ OS in the overall population stratified by stage in the TNM Classification of Malignant Tumors, 7th edition. DFS disease-free survival, RFS relapse-free survival, OS overall survival, 95\% CI 95\% confidence interval

in patients with stage II and III colon cancer such as JOIN, SACURA, and ACTS-CC trials was substantially lower than that in the NSABP C-07 trial, which enrolled subjects during the same period (17-26\% vs. $41 \%$, respectively). However, recently, a comparison of Japanese D3 lymph-node dissection and European complete mesocolic excision (CME) with central vascular ligation has been reported [24], and favorable outcomes of CME compared with conventional Westernstyle colon resection have also been reported from Western countries [25]. These findings imply that the gap in the survival of patients with stage II/III colon cancer between Japan and Western countries is closing, suggesting that Japanese outcomes might be applicable to the West. Considering the recent improved survival rate of patients with stage II/III colon cancer worldwide, we should reconsider the relatively high oxaliplatin-associated toxicity which could be weighed against the expectation of lower absolute treatment benefit in patients with a low risk of recurrence.

Recent IDEA collaboration reported 3 months adjuvant chemotherapy significantly reduced the rate of any grade PSN, compared with 6 months, without compromising efficacy in patients with low-risk stage III colon cancer $[26,27]$. In addition, the ACHIEVE trial, the part of IDEA collaboration, reported the incidence of any grade PSN lasting for 3 years was significantly lower for CAPOX (capecitabine plus oxaliplatin) than mFOLFOX6, suggesting that 3 months of CAPOX therapy may be the most appropriate option in low-risk patients [28], although ACHIEVE results need to be interpreted within the IDEA combined analysis as well as in terms of the reproducibility of the results across all trials. Therefore, 6 months of FOLFOX treatment even in the post-IDEA collaboration era is a standard adjuvant treatment in patients with curatively resected stage II/III colon cancer [3]. As the efficacy and safety of 6-month adjuvant mFOLFOX6 in Japanese patients with stage II/III colon cancer was confirmed in the JOIN trial, we moved forward to conduct two-phase three trials called the ACHIEVE trial for stage III and the ACHIEVE-2 trial for high-risk stage II [29, 30], which led to the first Japanese participation in the IDEA Collaboration $[26,27]$. 
Table 2 Multivariate analysis of risk factors for DFS, RFS and OS

\begin{tabular}{|c|c|c|c|c|c|c|c|c|c|}
\hline \multirow[b]{2}{*}{ Risk factor } & \multicolumn{3}{|l|}{ DFS } & \multicolumn{3}{|l|}{ RFS } & \multicolumn{3}{|l|}{ OS } \\
\hline & $\mathrm{HR}^{\mathrm{a}}$ & $95 \% \mathrm{CI}^{\mathrm{b}}$ & $P$ & HR & $95 \% \mathrm{CI}$ & $P$ & HR & $95 \% \mathrm{CI}$ & $P$ \\
\hline \multicolumn{10}{|l|}{$T$} \\
\hline SM, MP, SS, A & 1.000 & & & 1.000 & & & 1.000 & & \\
\hline SE, SI, AI & 1.386 & $1.041-1.845$ & 0.0255 & 1.441 & $1.073-1.937$ & 0.0152 & 1.406 & $0.878-2.251$ & 0.1556 \\
\hline \multicolumn{10}{|l|}{ Histology } \\
\hline Pap, tub & 1.000 & & & 1.000 & & & 1.000 & & \\
\hline Por, muc, sig & 1.570 & $1.028-2.398$ & 0.0367 & 1.569 & $1.017-2.419$ & 0.0416 & 2.860 & $1.619-5.051$ & 0.0003 \\
\hline \multicolumn{10}{|l|}{$N^{c}$} \\
\hline NO & 1.000 & & & 1.000 & & & 1.000 & & \\
\hline$N 1$ & 1.793 & $0.784-4.100$ & 0.1663 & 1.375 & $0.522-3.618$ & 0.5194 & 1.395 & $0.348-5.592$ & 0.6380 \\
\hline$N 2, N 3$ & 1.624 & $0.658-4.008$ & 0.2924 & 1.309 & $0.464-3.693$ & 0.6104 & 1.412 & $0.306-6.525$ & 0.6587 \\
\hline \multicolumn{10}{|l|}{$\mathrm{v}$} \\
\hline v0 & 1.000 & & & 1.000 & & & 1.000 & & \\
\hline v1, v2, v3 & 1.347 & $0.954-1.903$ & 0.0907 & 1.472 & $1.017-2.129$ & 0.0402 & 1.957 & $1.032-3.777$ & 0.0398 \\
\hline \multicolumn{10}{|l|}{$\mathrm{LN}$ ratio $(\%)$} \\
\hline $1-4$ & 1.000 & & & 1.000 & & & 1.000 & & \\
\hline $5-11$ & 1.469 & $0.719-3.001$ & 0.2918 & 2.011 & $0.855-4.732$ & 0.1094 & 1.326 & $0.382-4.607$ & 0.6572 \\
\hline $12-22$ & 1.920 & $0.938-3.930$ & 0.0742 & 2.741 & $1.165-6.444$ & 0.0208 & 1.563 & $0.446-5.483$ & 0.4854 \\
\hline $22<$ & 2.924 & $1.380-6.198$ & 0.0051 & 3.930 & $1.619-9.539$ & 0.0025 & 2.838 & $0.762-10.56$ & 0.1199 \\
\hline \multicolumn{10}{|l|}{ Tumor location } \\
\hline Right-sided & & & & & & & 1.000 & & \\
\hline Left-sided & & & & & & & 0.633 & $0.398-1.006$ & 0.0530 \\
\hline
\end{tabular}

${ }^{\mathrm{a}}$ Hazard ratio

${ }^{\mathrm{b}}$ Confidence interval

${ }^{\mathrm{c}}$ Japanese classification of colorectal carcinoma 7 th ed

Fig. 4 Recovery status of PSN during follow-up periods. PSN peripheral sensory neuropathy, trt treatment

\section{Recovery of PSN during follow-up periods}

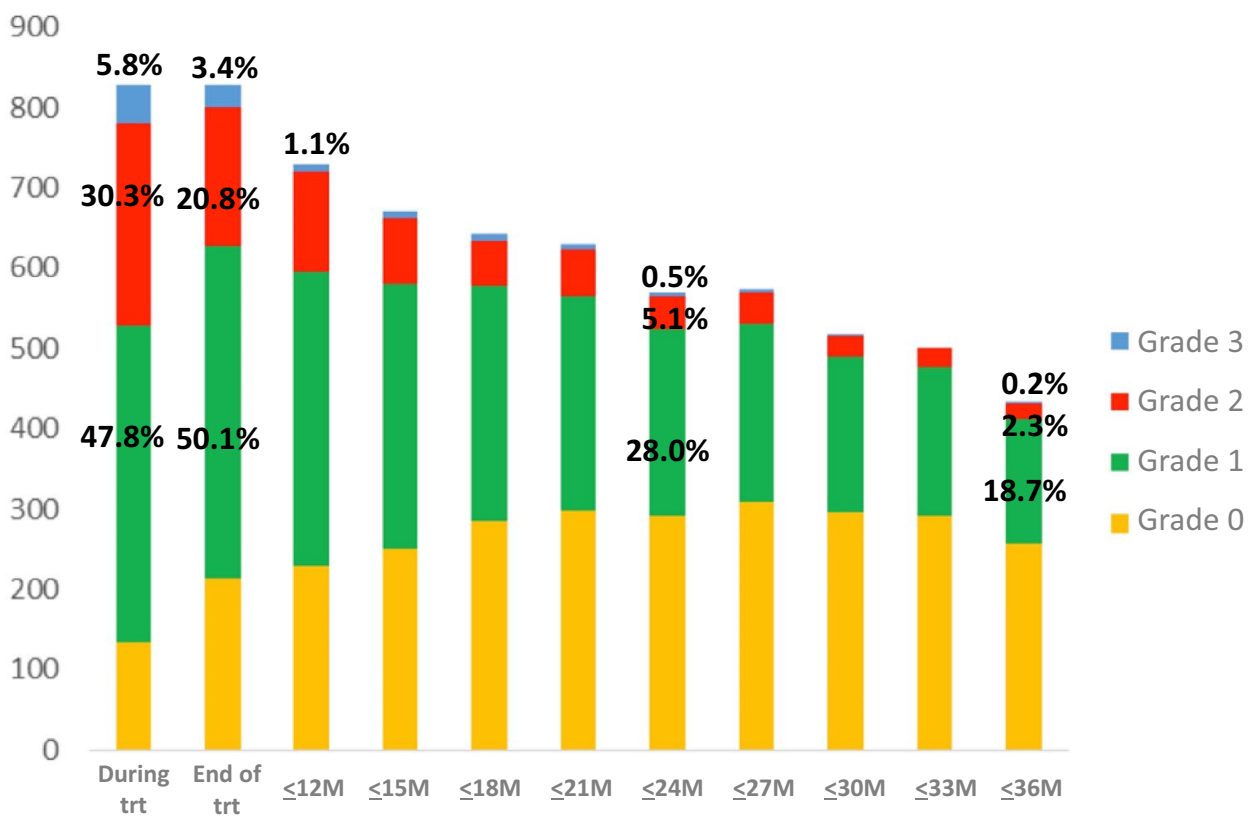




\section{Conclusion}

Six-month adjuvant mFOLFOX6 in patients with stage II/ III colon cancer is effective and safe. Most patients successfully recovered from grade 3 PSN related to oxaliplatin, but approximately $20 \%$ of patients had grade 1 or grade 2 PSN at the 3-year follow-up.

Acknowledgements We thank the patients who participated in this study and their families, the physicians and medical staff of the 198 institutions registered in this study, Statcom Co., Ltd., for performing statistical analysis of the data, and the JFMC.

Funding The trial was conducted by the JFMC, a non-commercial organization for investigator-initiated cancer trials, and funded by Yakult Honsha Co., Ltd. under contract. Yakult Honsha played no role in the design, collection, analysis or interpretation of the data, or writing of this manuscript.

\section{Compliance with ethical standards}

Conflict of interest T. Yoshino has received grants from MSD K.K., Sanofi K.K., Sumitomo Dainippon Pharma Co., Ltd., Chugai Pharmaceutical Co., Ltd., GlaxoSmithKline K.K., and Nippon Boehringer Ingelheim Co., Ltd., and honoraria from Sanofi K.K., Chugai Pharmaceutical Co., Ltd., Eli Lilly Japan K.K, and Merck Serono Co., Ltd. outside the submitted work; M. Kotaka has received honoraria from Chugai Pharmaceutical Co., Ltd., Yakult Honsha Co., Ltd., Takeda Pharmaceutical Co., Ltd., Merck Serono Co., Ltd., and Taiho Pharmaceutical Co., Ltd. outside the submitted work; K. Shinozaki has received honoraria from Chugai Pharmaceutical Co., Ltd., Takeda Pharmaceutical Co., Ltd., Mochida Pharmaceutical Co., Ltd., Merck Serono Co., Ltd., Taiho Pharmaceutical Co., Ltd., Yakult Honsha Co., Ltd., Astellas Pharma Inc., Novartis Pharma K.K., Eisai Co., Ltd., Eli Lilly Japan K.K., Shionogi \& Co., Ltd., Kyowa Hakko Kirin Co., Ltd., and Asahi Kasei Pharma Co. outside the submitted work; T. Touyama declares no conflicts of interest; D. Manaka declares no conflicts of interest; T. Matsui declares no conflicts of interest; K. Ishigure has received honoraria from ONO Pharmaceutical Co., Ltd., Takeda Pharmaceutical Co., Ltd., Chugai Pharmaceutical Co., Ltd., and Yakult Honsha Co., Ltd. outside the submitted work; J. Hasegawa declares no conflicts of interest; K. Inoue declares no conflicts of interest; Y. Munemoto declares no conflicts of interest; A. Takagane declares no conflicts of interest; $H$. Ishikawa declares no conflicts of interest; H. Ishida has received personal fees from Asahi Kasei, Takeda Pharmaceutical Co., Ltd., and Chugai Pharmaceutical Co., Ltd. outside the submitted work; Y. Ogata declares no conflicts of interest; K. Oba has received personal fees from Eisai Co., Ltd., Bristol-Myers Squibb K.K., Merck Serono Japan, ONO Pharmaceutical Co., Ltd., Asahi Kasei, Takeda Pharmaceutical Co., Ltd., Daiichi Sankyo, Inc. and Chugai Pharmaceutical Co., Ltd. outside the submitted work; K. Goto has received grants and personal fees from Stemcentrx (now part of AbbVie), AstraZeneca K.K., Boehringer Ingelheim, Bristol-Myers Squibb K.K., Chugai Pharmaceutical Co., Ltd., Daiichi Sankyo, Inc., Eli Lilly Japan K.K., Life Technologies Japan Ltd., Merck Serono Co., Ltd., MSD K.K., Novartis Pharma K.K., ONO Pharmaceutical Co., Ltd., Pfizer Japan Inc., Riken Genesis Co., Ltd., Taiho Pharmaceutical Co., Ltd., and Takeda Pharmaceutical Co., Ltd., grants from Amgen Astellas BioPharma K.K., Astellas Pharma Inc., Eisai Co., Ltd., Ignyta Inc., Janssen Pharmaceutical K.K., Kyowa Hakko Kirin Co., Ltd., Loxo Oncology, Inc., Oxonc, RTI Health Solutions, Sumitomo Dainippon Pharma Co., Ltd., as well as personal fees from F. Hoffmann-La Roche Ltd., Nippon Kayaku Co., Ltd., Otsuka Pharmaceutical Co.,
Ltd., and SRL Inc. outside the submitted work; J. Sakamoto has received consultant fees from Takeda Pharmaceutical Co., Ltd., honoraria from Tsumura Co., Ltd., Nippon Kayaku Co., Ltd., and Chugai Pharmaceutical Co., Ltd. outside the submitted work; Y. Maehara has received grants from Yakult Honsha Co., Ltd. outside the submitted work; A. Ohtsu has received grants and personal fees from Bristol-Myers Squibb K.K., personal fees from ONO Pharmaceutical Co., Ltd., Chugai Pharmaceutical Co., Ltd., and Taiho Pharmaceutical Co., Ltd. outside the submitted work.

Ethical approval The institutional review board at each study center approved the protocol, and the study was conducted by the Japanese Foundation for Multidisciplinary Treatment of Cancer (JFMC), a non-commercial organization for investigator-initiated cancer trials, in accordance with the principles expressed in the Declaration of Helsinki. All patients provided written informed consent prior to enrolment. This study is registered with UMIN Clinical Trial Registry (Trial Identifier UMIN000004443).

Open Access This article is distributed under the terms of the Creative Commons Attribution 4.0 International License (http://creativeco mmons.org/licenses/by/4.0/), which permits unrestricted use, distribution, and reproduction in any medium, provided you give appropriate credit to the original author(s) and the source, provide a link to the Creative Commons license, and indicate if changes were made.

\section{References}

1. Labianca R, Nordlinger B, Beretta GD et al (2013) Early colon cancer: ESMO clinical practice guidelines for diagnosis, treatment and follow-up. Ann Oncol 24(Suppl 6):vi64-vi72

2. Watanabe T, Muro K, Ajioka Y et al (2017) Japanese society for cancer of the colon and rectum (JSCCR) guidelines 2016 for the treatment of colorectal cancer. Int J Clin Oncol 23:1

3. NCCN guidelines for patients: colon cancer version 4 (2018) www.nccn.org. Accessed 5 Nov 2018

4. Andre T, Boni C, Mounedji-Boudiaf L et al (2004) Oxaliplatin, fluorouracil, and leucovorin as adjuvant treatment for colon cancer. N Engl J Med 350:2343-2351

5. Andre T, Boni C, Navarro M et al (2009) Improved overall survival with oxaliplatin, fluorouracil, and leucovorin as adjuvant treatment in stage II or III colon cancer in the MOSAIC trial. J Clin Oncol 27:3109-3116

6. Lee P-H, Park Y-S, Ji J-F, Fu Y-T, Ratanatharathorn V (2009) Safety and tolerability of FOLFOX4 in the adjuvant treatment of colon cancer in Asian patients: the MASCOT study. Asia-Pac J Clin Oncol 5:101-110

7. Allegra CJ, Yothers G, O'Connell MJ et al (2009) Initial safety report of NSABP C-08: a randomized phase III study of modified FOLFOX6 with or without bevacizumab for the adjuvant treatment of patients with stage II or III colon cancer. J Clin Oncol 27:3385-3390

8. Allegra CJ, Yothers G, O'Connell MJ et al (2011) Phase III trial assessing bevacizumab in stages II and III carcinoma of the colon: results of NSABP protocol C-08. J Clin Oncol 29:11-16

9. Kotaka M, Yoshino T, Oba K et al (2015) Initial safety report on the tolerability of modified FOLFOX6 as adjuvant therapy in patients with curatively resected stage II or III colon cancer (JFMC41-1001-C2: JOIN trial). Cancer Chemother Pharmacol 76:75-84

10. Kidwell KM, Yothers G, Ganz PA et al (2012) Long-term neurotoxicity effects of oxaliplatin added to fluorouracil and leucovorin 
as adjuvant therapy for colon cancer: results from National Surgical Adjuvant Breast and Bowel Project trials C-07 and LTS-01. Cancer 118:5614-5622

11. Mols F, Beijers T, Lemmens V, van den Hurk CJ, Vreugdenhil G, van de Poll-Franse LV (2013) Chemotherapy-induced neuropathy and its association with quality of life among 2- to 11-year colorectal cancer survivors: results from the population-based PROFILES registry. J Clin Oncol 31:2699-2707

12. Pachman DR, Qin R, Seisler DK et al (2015) Clinical course of oxaliplatin-induced neuropathy: results from the randomized phase III trial N08CB (alliance). J Clin Oncol 33:3416-3422

13. Pietrangeli A, Leandri M, Terzoli E, Jandolo B, Garufi C (2006) Persistence of high-dose oxaliplatin-induced neuropathy at longterm follow-up. Eur Neurol 56:13-16

14. Tofthagen C, Donovan KA, Morgan MA, Shibata D, Yeh Y (2013) Oxaliplatin-induced peripheral neuropathy's effects on healthrelated quality of life of colorectal cancer survivors. Support Care Cancer 21:3307-3313

15. Land SR, Kopec JA, Cecchini RS, Ganz PA, Wieand HS, Colangelo LH, Murphy K, Kuebler JP, Seay TE, Needles BM, Bearden JD 3rd, Colman LK, Lanier KS, Pajon ER Jr, Cella D, Smith RE, O'Connell MJ, Costantino JP, Wolmark N (2007) Neurotoxicity from oxaliplatin combined with weekly bolus fluorouracil and leucovorin as surgical adjuvant chemotherapy for stage II and III colon cancer: NSABP C-07. J Clin Oncol 25(16):2205-2211 (Epub 2007 Apr 30)

16. Kotani D, Kuboki Y, Yoshino T (2016) Adjuvant chemotherapy for colon cancer: guidelines and clinical trials in Japan. Curr Colorectal Cancer Rep 12:289-295

17. Kondo K, Sadahiro S, Tsuchiya T, Sasaki K, Katsumata K, Nishimura G, Kakeji Y, Baba H, Kodaira S, Saji S (2012) Phase III trial of treatment duration for oral uracil and tegafur/leucovorin adjuvant chemotherapy for patients with stage IIB/III colon cancer: results of JFMC33-0502. ESMO 2012 Abstract \#552P

18. Yamaguchi S, Kunieda K, Sato T, Naomoto Y, Kobayashi M, Ogata Y, Furuhata T, Takii Y, Kusunoki M, Maehara Y, Koda K, Okuno K, Ohno M, Mishima H, Sadahiro S, Hamada C, Sakamoto J, Saji S, Tomita N (2016) Phase III trial of 24 weeks vs. 48 weeks capecitabine adjuvant chemotherapy for patients with stage III colon cancer: final results of JFMC37-0801. ESMO 2016 Abstract \#469PD

19. Yoshida M, Ishiguro M, Ikejiri K, Mochizuki I, Nakamoto Y, Kinugasa Y, Takagane A, Endo T, Shinozaki H, Takii Y, Mochizuki H, Kotake K, Kameoka S, Takahashi K, Watanabe T, Watanabe M, Boku N, Tomita N, Nakatani E, Sugihara K (2014) ACTSCC study group. S-1 as adjuvant chemotherapy for stage III colon cancer: a randomized phase III study (ACTS-CC trial). Ann Oncol 25(9): 1743-1749

20. Shimada Y, Hamaguchi T, Mizusawa J, Saito N, Kanemitsu Y, Takiguchi N, Ohue M, Kato T, Takii Y, Sato T, Tomita N, Yamaguchi S, Akaike M, Mishima H, Kubo Y, Nakamura K, Fukuda H, Moriya Y (2014) Randomised phase III trial of adjuvant chemotherapy with oral uracil and tegafur plus leucovorin versus intravenous fluorouracil and levofolinate in patients with stage III colorectal cancer who have undergone Japanese D2/D3 lymph node dissection: final results of JCOG0205. Eur J Cancer 50(13):2231-2240. https://doi.org/10.1016/j.ejca.2014.05.025 (Epub 2014 Jun 21)

21. Hamaguchi T, Shimada Y, Mizusawa J, Kinugasa Y, Kanemitsu Y, Ohue M, Fujii S, Takiguchi N, Yatsuoka T, Takii Y, Ojima H, Masuko H, Kubo Y, Mishima H, Yamaguchi T, Bando H, Sato T,
Kato T, Nakamura K, Fukuda H, Moriya Y (2018) Capecitabine versus $\mathrm{S}-1$ as adjuvant chemotherapy for patients with stage III colorectal cancer (JCOG0910): an open-label, non-inferiority, randomised, phase 3, multicentre trial. Lancet Gastroenterol Hepatol $3(1): 47-56$

22. Kajiwara Y, Ishiguro M, Teramukai S, Matsuda C, Fujii S, Kinugasa Y, Nakamoto Y, Kotake M, Sakamoto Y, Kurachi K, Maeda A, Komori K, Tomita N, Shimada Y, Takahashi K, Kotake K, Watanabe M, Mochizuki H, Sugihara K, SACURA Study Group (2018) A randomized phase III trial of 1-year adjuvant chemotherapy with oral tegafur-uracil (UFT) vs surgery alone in stage II colon cancer: SACURA trial. Eur J Cancer 96:54 (ASCO2016 Abstract \#3617)

23. Chang GJ, Rodriguez-Bigas MA (2007) Lymph node evaluation and survival after curative resection of colon cancer: systematic review. J Natl Cancer Inst 99(6):433-441

24. Nicholas PW, Kobayashi H, Takahashi K et al (2012) Understanding optimal colonic cancer surgery: comparison of Japanese D3 resection and European complete mesocolic excision with central vascular ligation. J Clin Oncol 30(15):1763-1769

25. Bertelsen CA, Neuenschwander AU, Jansen JE et al (2015) Disease-free survival after complete mesocolic excision compared with conventional colon cancer surgery: a retrospective, population-based study. Lancet Oncol 16(2):161-168

26. Andre T, Iveson T, Labianca R et al (2013) The IDEA (International Duration Evaluation of Adjuvant Chemotherapy) collaboration: prospective combined analysis of phase III trials investigating duration of adjuvant therapy with the FOLFOX (FOLFOX4 or Modified FOLFOX6) or XELOX (3 versus 6 months) regimen for patients with stage III Colon Cancer: trial design and current status. Curr Colorectal Cancer Rep 9:261-269

27. Grothey A, Sobrero AF, Shields AF et al (2018) Duration of adjuvant chemotherapy for stage III colon cancer. N Engl J Med 378(13):1177-1188

28. Takeuchi S, Yoshino T, Yamanaka T et al (2018) Long-term effect of peripheral sensory neuropathy (PSN) of 3 or 6 months oxaliplatin-based adjuvant chemotherapy for stage III colon cancer: ACHIEVE as part of the IDEA collaboration. Age 70:70 (WCGC Abstract \#O-028)

29. Yoshino T, Watanabe T, Mori M, et al (2014) Two phase III studies comparing 6 months of either mFOLFOX6 or XELOX with 3 months of the same regimen as adjuvant chemotherapy in patients with completely resected stage III colon cancer (ACHIEVE) or high-risk stage II colon cancer (ACHIEVE-2). ASCO 2014 Abstract \#TPS 3655

30. Yoshino T, Yamanaka T, Kotaka M, et al (2017) Efficacy of 3 versus 6 months of oxaliplatin-based adjuvant chemotherapy for stage III colon cancer (CC): Results from phase III ACHIEVE trial as part of the International Duration Evaluation of Adjuvant therapy (IDEA) collaboration. ESMO 2017 Abstract \#LBA 24

Publisher's Note Springer Nature remains neutral with regard to jurisdictional claims in published maps and institutional affiliations. 


\section{Affiliations}

Takayuki Yoshino $^{1} \cdot$ Masahito Kotaka $^{2} \cdot$ Katsunori Shinozaki $^{3} \cdot$ Tetsuo Touyama $^{4} \cdot$ Dai Manaka $^{5} \cdot$ Takanori Matsui $^{6}$. Kiyoshi Ishigure $^{7} \cdot$ Junichi Hasegawa $^{8} \cdot$ Keiji Inoue $^{9} \cdot$ Yoshinori Munemoto $^{10} \cdot$ Akinori Takagane $^{11}$. Hiroshi Ishikawa ${ }^{12} \cdot$ Hideyuki Ishida $^{13} \cdot$ Yutaka Ogata $^{14} \cdot$ Koji Oba $^{15} \cdot$ Koichi Goto $^{16}$. Junichi Sakamoto ${ }^{17}$. Yoshihiko Maehara ${ }^{18} \cdot$ Atsushi Ohtsu ${ }^{1}$

1 Department of Gastroenterology and Gastrointestinal Oncology, National Cancer Center Hospital East, 6-5-1 Kashiwanoha, Kashiwa 277-8577, Japan

2 Gastrointestinal Cancer Center, Sano Hospital, Kobe 655-0031, Japan

3 Division of Clinical Oncology, Hiroshima Prefectural Hospital, Hiroshima 734-8530, Japan

4 Department of Surgery, Nakagami Hospital, Okinawa 904-2195, Japan

5 Department of Surgery, Gastrointestinal Center, Kyoto Katsura Hospital, Kyoto 615-8256, Japan

6 Department of Gastroenterological Surgery, Aichi Cancer Center Aichi Hospital, Aichi 444-0011, Japan

7 Department of Surgery, Konan Kosei Hospital, Konan, Aichi 483-8704, Japan

8 Department of Surgery, Osaka Rosai Hospital, Osaka 591-8025, Japan

9 Surgery Nagasaki Harbor Medical Center City Hospital, Nagasaki 850-8555, Japan

10 Department of Surgery, Fukui-ken Saiseikai Hospital, Fukui 918-8503, Japan
11 Department of Surgery, Hakodate Goryoukaku Hospital, Hokkaido 040-8611, Japan

12 Department of Gastrointestinal Surgery, Sasebo City General Hospital, Nagasaki 857-8511, Japan

13 Department of Digestive Tract and General Surgery, Saitama Medical Center, Saitama Medical University, Kawagoe 350-8550, Japan

14 Cancer Center, Kurume University Hospital, Fukuoka 830-0011, Japan

15 Department of Biostatistics, School of Public Health, Graduate School of Medicine, and Interfaculty Initiative in Information Studies, The University of Tokyo, Tokyo 113-8655, Japan

16 Department of Thoracic Oncology, National Cancer Center Hospital East, Kashiwa 277-8577, Japan

17 Tokai Central Hospital, Kakamigahara 504-8601, Japan

18 Japanese Foundation for Multidisciplinary Treatment of Cancer, Tokyo 136-0071, Japan 